\title{
Validation of the direct numerical simulation code based on the criteria of force coefficient convergence
}

\author{
Z. B. Jiang, L. Z. Shao \& F. Shao \\ PLA University of Science \& Technology, China
}

\begin{abstract}
The open computational fluid dynamics (CFD) code called Gerris is very popular recently for its grid discretising strategy using quad/octree. It is available to numerically simulate a large range of problems in fluid domains and the simulation preprocess of grid is easy to represent the complex solid boundary by means of volume of fluid (VOF) approach.

Two classical two-dimensional (2D) bluff and streamlined bodies, namely circle cylinder and airfoil NACA2414, are simulated firstly with various Reynolds numbers and grid resolutions. The DNS results in this paper are validated based on the criteria of the force coefficient convergence with various grid levels. The three-dimensional (3D) floating sphere and series 60 ship hull are also simulated including the water free surface and induced wave with various Froude numbers and the results are validated employing the same previous processes.

The results show that the force coefficients acted on the bluff bodies without water surface cannot easily converge to steady ones even with fine grids of high resolutions. Comparatively, the force coefficient of the streamlined body without water free surface is easier to converge even with turbulent flow. The results of 3D computational cases with water free surface show that the force coefficients would converge to desirable ones with grid level 8 and 9 both for the series 60 ship hull and the floating sphere, which are bluff body and streamlined body, respectively. Generally, Gerris is very suitable for calculating the cases with water free surface with relative low grid resolution.
\end{abstract}

Keywords: computational fluid dynamics, Gerris, direct numerical simulation, volume of fluid, bluff body, streamlined body. 


\section{Introduction}

Gerris is one of computer codes in the field of computational fluid dynamics (CFD). It was released as free and open-source software, subjected to the requirements of the GNU General Public License (GPL) [1] and it is very popular recently for its outstanding peculiarities for solving a large scope of scientific and engineering problems involving fluid. Gerris solves the Navier-Stokes (NS) equations in two or three dimensions, allowing to model wind erosion [2], free water surface (like as shallow water wave $[3,4]$, solitary wave $[5,6]$ and tsunami [7,8]), industrial fluids [9], droplets [10], even Bingham plastic fluid [11] and granular column [12,13], etc.

Generally, the flow flied is mathematically modelled by partial differential equations, i.e., NS equations and they cannot be solved analytically for a majority of the flow problems. However, the numerical solutions can be obtained with the advent of the modern computers. Several methods can be used to provide numerical solutions to partial differential equations, i.e.,finite differences, finite volumes and finite elements, and Gerris belongs to the finite volumes family of CFD models [1].

Most models use meshes which are either structured (Cartesian or curvilinear grids) or unstructured (triangular, tetrahedral, etc.). Gerris is quite different on this respect: it implements a deal between structured and unstructured meshes by using a tree data structure $[1,10,14]$, allowing to refine locally (and dynamically) the finite-volume description of the pressure and velocity fields. Indeed the grid evolves in the course of a given simulation owing to criteria defined by the user (e.g., dynamic refinement of the grid in the vicinity of sharp gradients). Gerris mainly aims at DNS and the fluid flow turbulence characteristics can be well simulated and they were well agreed with experiments around a research vessel [15].

Gerris can predict time evolutions of flow velocities, pressures and free surfaces with appreciable precisions. However, it is not the case to predict the force coefficient on the solid bodies embedded in the flow fields. The reason is that the quadtree/actree grid is employed in this simulation code to approximate the solid boundaries and it is not enough precise to represent the bluff body boundaries, even with a large number of numerical grids.

In this paper, Gerris code is employed to predict the force coefficients on the bluff or streamlined bodies with or without water free surface and the criteria of force coefficient convergence is employed to determine the qualities of the simulation results. The remaining of this paper is organized as follows. The numerical methods including governing equations, solver scheme and grid strategy are represented briefly in section 2 . The numerical simulation results are shown in section 3 and the results containing the flow fields and the force coefficient on the bluff body and the streamlined body with or without free water surface. The 2D circular cylinder and the airfoil NACA2414 are simulated without water surface, and the 3D floating sphere and series 60 ship hull are computed with water surface. The conclusions of all simulated cases are drawn in section 4 and some 
useful suggestions are listed for the using of Gerris involving the forces on the solid bodies in the flow field with direct numerical simulation.

\section{Numerical method}

The numerical model was setup using the Gerris Flow Solver [1] and it is a 2D/3D equation solver designed to solve the continuity equation and the incompressible momentum equation, i.e., NS equations, like as

$$
\begin{gathered}
\nabla \cdot \mathbf{u}=\mathbf{0} \\
\rho\left(\frac{\partial \mathbf{u}}{\partial t}+\mathbf{u} \cdot \nabla \mathbf{u}\right)=-\nabla p+\nabla \cdot\left(\mu\left(\nabla \mathbf{u}+\nabla^{\mathbf{T}} \mathbf{u}\right)\right)
\end{gathered}
$$

where $\mathbf{u}$ is the velocity vector, $p$ the pressure, $\rho$ the fluid density and $\mu$ the dynamic viscosity of the fluid. Among its many features, the code is based around a 2D quadtree or 3D octree finite-volume discretisation of the NS equations [1, 10, 14]. The quadtree or octree mesh notably allows easy handling of non-uniformly refined meshes. Mesh refinement is adaptive in space and in time and can be setup using geometrical criteria as well as criteria based on the computed quantities.

As mentioned above, the finite volume method is used to solve the set of equations of the continuity equation and the incompressible NS equations. The adaptive grid technique is used in the computation, and the domain is spatially discreted using cubic finite volumes organised hierarchically as an quadtree/octree. The projection method and multi-grid method are used to solve the pressure equation. The convective term is discreted with second order upwind schemes, and the volume of fluid (VOF) method is applied to treat free surface boundaries [1].

A 2D example of spatial discretisation and the corresponding tree representation is represented here, as shown in Figure 1. The length of a finite volume (cell) edge is denoted by $h$. Each cell may be the parent of up to four children. The root cell is the base of the tree and a leaf cell is a cell without any child. The level of a cell is defined by starting from zero for the root cell and by adding one every time a group of four descendant children is added. Each cell $\mathcal{C}$ has a direct neighbour at the same level in each direction $d$, noted $\mathcal{N}_{d}$. Each of these neighbours is accessed through a face of the cell, noted $\mathcal{C}_{d}$. In order to handle embedded solid boundaries, we also define mixed cells which are cut by a solid boundary. The primitive variables of the NS equations (velocity $\mathbf{u}$ and pressure $p$ ) are all defined at the centre of the cells.

\section{Numerical results}

The classical 2D cylinder and airfoil NACA2414 flows are numerically simulated with DNS to test the precisions of the Gerris code and the relationship between the force coefficient convergence and the grid resolutions. Gerris uses the concept of grid box in the preprocess and the flow domains are combined with certain boxes connected by edges. The fully computational grids for the cylinder and the airfoil 

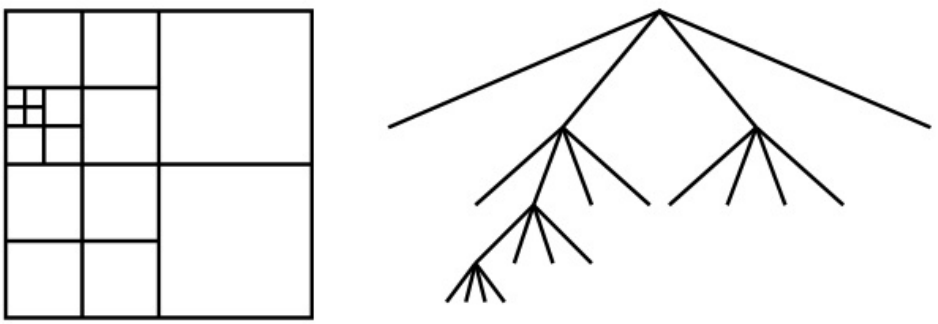

Figure 1: Quadtree discretisation (left) and corresponding logical structure (right).

are shown in Figure 2. The whole computational domain has eight boxes and the grids are adaptive just as described below and they are visually shown in Figure 2 of (a) cylinder and (b) airfoil, respectively, after 10 nondimensional time.

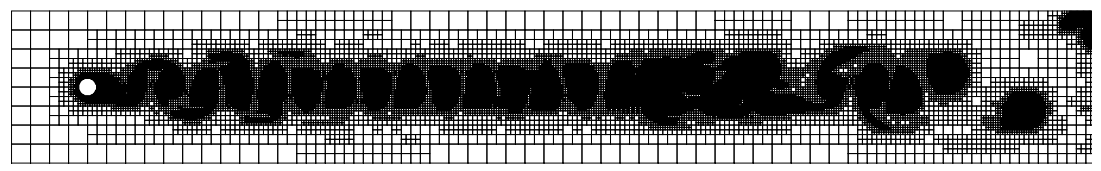

(a) Cylinder

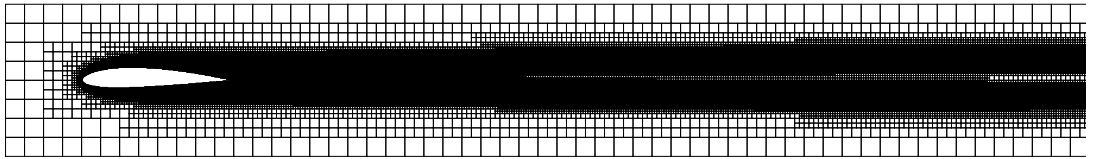

(b) Airfoil NACA2414

Figure 2: Adaptive grid of the computational domain.

The adaptive grid is applied in this computation. The adaptive refinement strategy includes two steps. In the first step, all the leaf cells which satisfy a given criterion are refined. In a second step, we consider the parent cells of all the leaf cells. All of these cells which satisfy the refinement criterion are refined and which do not satisfy the refinement criterion would be coarsened. The criterion is based on the norm of the local vorticity vector, like as

$$
\frac{h\|\nabla \mathbf{u}\|}{\max \|\mathbf{u}\|}>\tau,
$$

where $h$ is the minimum of the grid size and $\tau$ is the threshold value of refinement.

similarly, the 3D computational grids of the series 60 ship hull and the floating sphere are shown in Figures 3 and 4, respectively. Two plane grids, horizontal plane coinciding with water free surface and vertical plane in the symmetry boundary, are presented after 8 nondimensional time. And again, the adaptive grids are clearly shown according to eqn (3). 


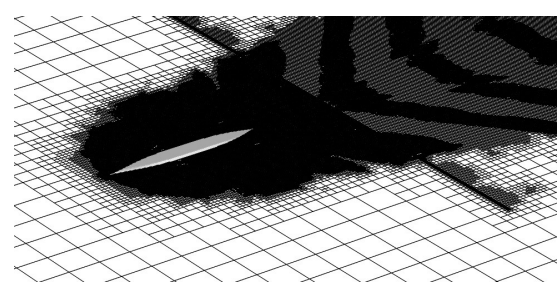

(a) Horizontal

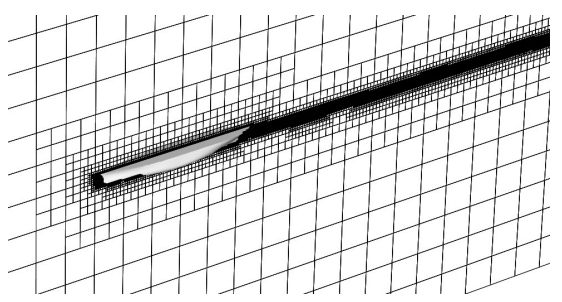

(b) Vertical

Figure 3: Adaptive grid of the ship hull.

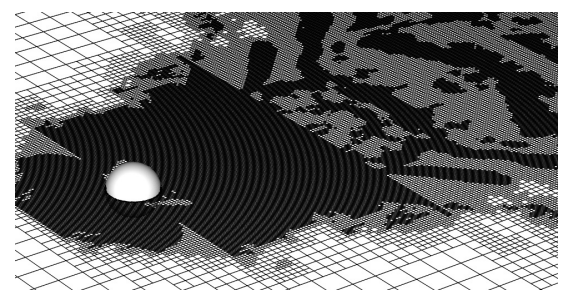

(a) Horizontal

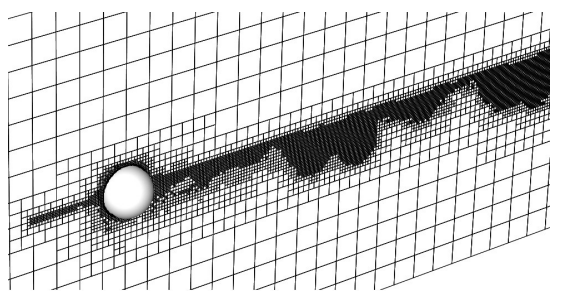

(b) Vertical

Figure 4: Adaptive grid of the floating sphere.

\subsection{Force variation and its convergence of 2D}

For 2D cylinder and airfoil, the computational cases are carried out with Reynolds number $R e=100, R e=1000, R e=1 \times 10^{4}$ and $R e=1 \times 10^{5}$. The Reynolds number is defined as

$$
R e=\frac{U L}{\nu},
$$

where $U$ is the inlet velocity of the fluid, $L$ is the characteristic length of the object, which is the diameter for cylinder and the chord length for airfoil, $\nu$ is the viscosity of the fluid. In this paper, all parameters are nondimensional and the inlet velocity is uniform and equal to 1 , and the characteristic lengths are 0.0625 for cylinder and 1 for airfoil. The values of viscosity are adjusted to meet different Reynolds numbers for various computational cases. All input values are nondimensional and it is easy for us to analysis the results based on certain nondimensional numbers, like as Reynolds number, lift and drag coefficients, etc.

The pressure fields and the vorticity fields are shown in Figures 5(a) and 6(a), Figures 5(b) and 6(b) with Reynolds number 100 and grid level 7, for cylinder and airfoil, respectively.

In Figure 6(a), the well-known Karman vortex street is generated and it is a very classical case for CFD. But the vortex street is not visual for the streamline body, e.g., the airfoil, with Reynolds number 100 in Figure 6(b). In this paper, the vortex street is not the focus to discuss. However, the various grid levels are calculated to 


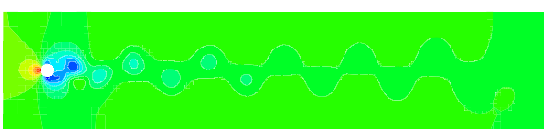

(a) Cylinder

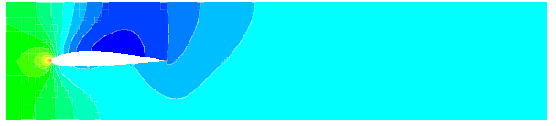

(b) Airfoil NACA2414

Figure 5: Pressure at $R e=100$ with grid level 7.

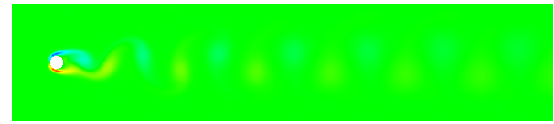

(a) Cylinder

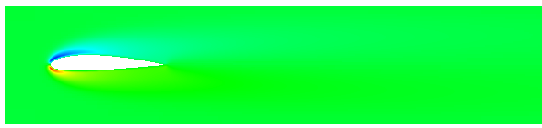

(b) Airfoil NACA2414

Figure 6: Vorticity at $R e=100$ with grid level 7 .

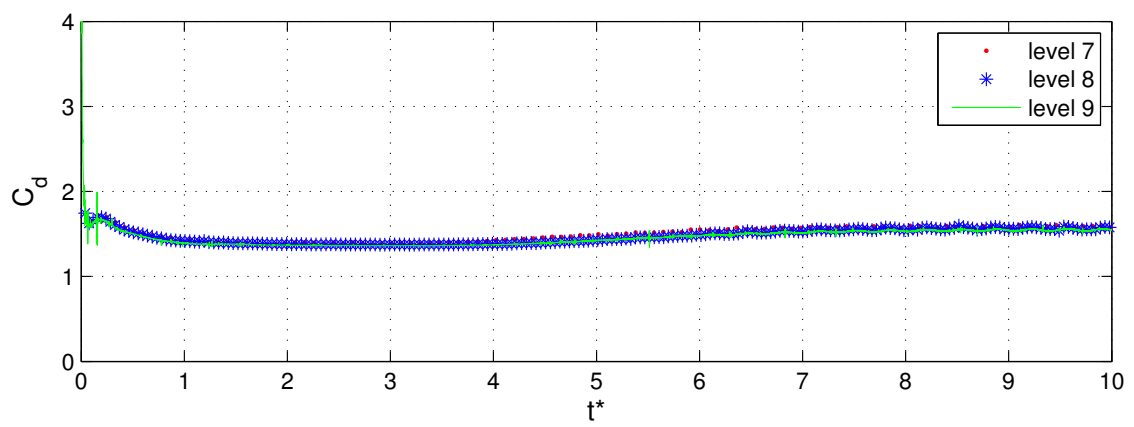

(a) Cylinder

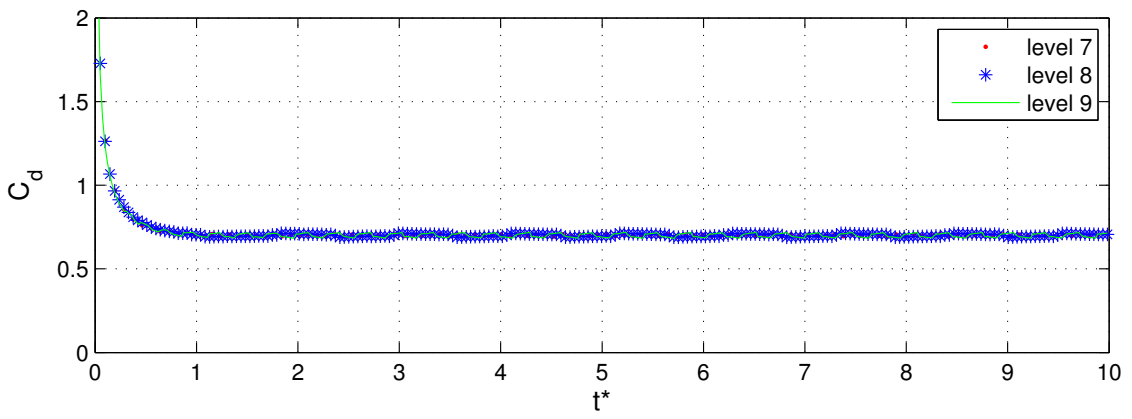

(b) Airfoil NACA2414

Figure 7: Drag force coefficient variations with nondimensional time at $R e=100$. 


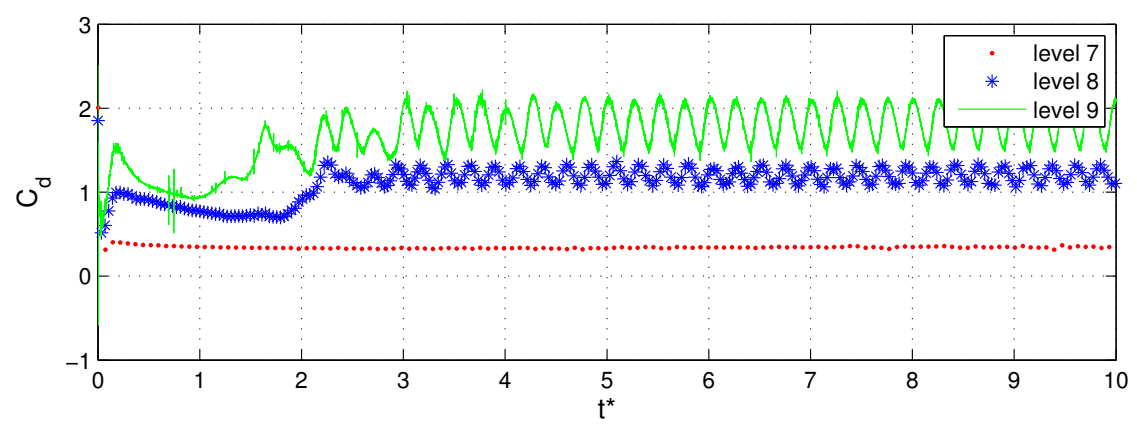

(a) Cylinder

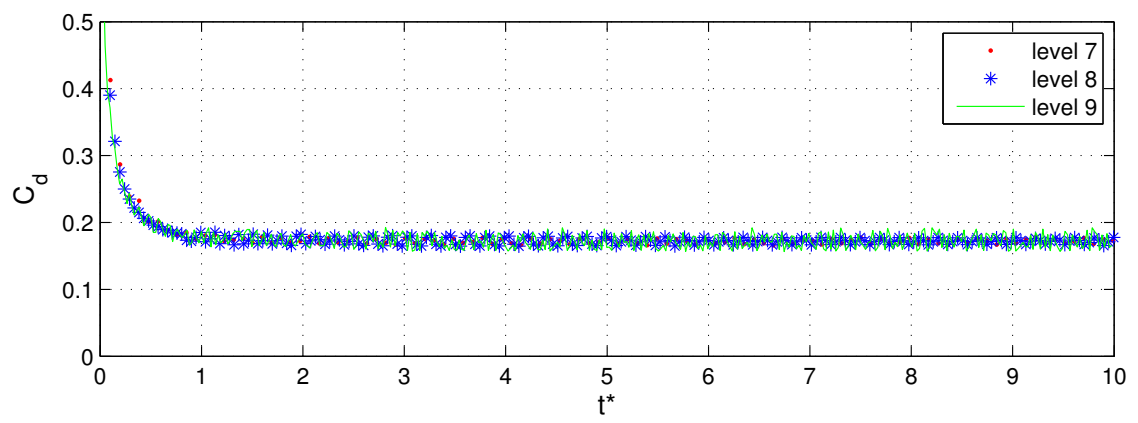

(b) Airfoil NACA2414

Figure 8: The drag force coefficient variations with time at $R e=1000$.

test the convergence of the results and how to judge the convergence is our main emphasis.

Three grid levels $L_{g}$ are calculated, e.g., $L_{g}=7,8$ and 9 for various Reynolds numbers mentioned above. The drag force coefficients are convergent with three different grid resolutions for both cylinder and airfoil flow fields, as shown in Figure 7, respectively. For cylinder with $R e=100$, the drag coefficient is about 1.57 , which is very close to the other's computational and experimental results [16].

The cylinder case has longer time convergent process and this case takes about 7 nondimensional time and after then a little fluctuation occurs. And the frequency of this fluctuation is coincident with that of the shedding of the vortex street. The shedding of the vortex street means that the flow field of the cylinder with $R e=100$ turns into turbulent status and the grid level 7 is enough for its convergence. The minimum grid size can be evaluated by

$$
h_{m}=\frac{1}{2^{L}}=\frac{1}{2^{7}}=\frac{1}{128}=0.0078 .
$$

For the airfoil case, it only takes about 0.5 nondimensional time to convergent and the force coefficient has no fluctuation. This means that the flow field of the 


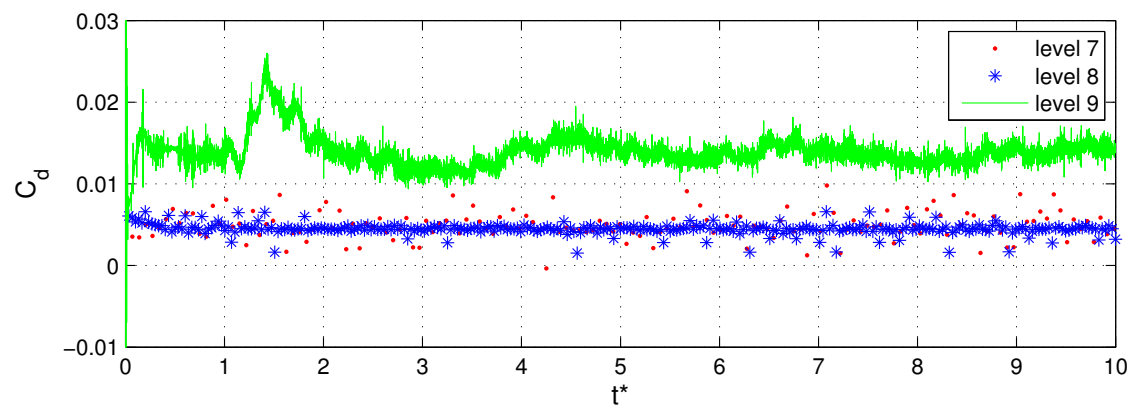

(a) Cylinder

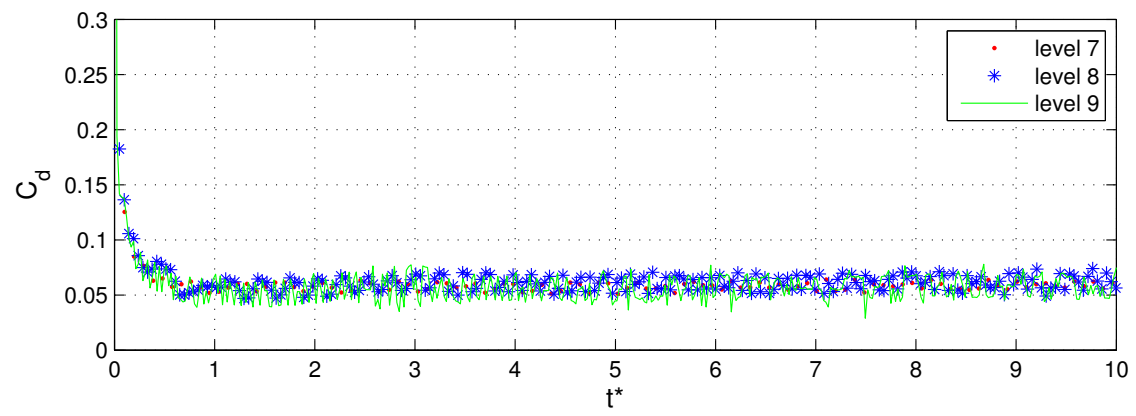

(b) Airfoil NACA2414

Figure 9: The drag force coefficient variations with time at $R e=1 \times 10^{4}$.

airfoil with $R e=100$ is laminar and the grid level 7 is enough for its convergence of both for velocity and pressure. The value of the drag coefficient is close to the other's computational result [17].

The drag force convergent processes with three different grid resolutions are shown in Figure 8 for both (a) cylinder and (b) airfoil with $R e=1000$. The fields of pressure and vorticity are not presented for the limitation of the paper length.

As shown in Figure 8, the drag coefficients of cylinder have much differences with different grid resolutions. So it means that the grid level even 9 is not enough for the cylinder case to get the force coefficient convergence. But for airfoil case, the drag coefficient is convergent even for grid level 7 , for three grid levels reach the same value after 0.5 seconds calculation.

The minimum grid size for grid level 9 is about

$$
h_{m}=\frac{1}{2^{L}}=\frac{1}{2^{9}}=\frac{1}{512}=0.0020 .
$$

For cylinder case, grid level 9 is suitable Reynolds number approximate $R e=500$, which is equal to $1 / h_{m}$ and it is not enough for $R e=1000$. But for airfoil case, the grid level 9 is good for its convergence of flow fields and force coefficients. 
In Figure 8(b), the drag coefficient has slight fluctuation because the flow field turns into turbulent status and the shedding of vortex exists.

The cases with $R e=1 \times 10^{4}$ are the same as that with $R e=1000$ for both cylinder and airfoil, as shown in Figure 9. However, there are more fluctuations because the turbulence will be more violent with higher Reynolds number.

But for the cases of both cylinder and airfoil, the drag coefficient would not convergent to stable values with $R e=1 \times 10^{5}$, as shown in Figure 10. The amplitudes of the fluctuations are so violent that the drag coefficients can not reflect the true tendency of convergence. Under this Reynolds number, the drag force convergence is hard to obtain, even for finer grid resolution, e.g., the minimum grid is about 0.002 , for streamlined object. In order to gain ideal results, finer grid is required to calculate. And the minimum grid is of the order of magnitude of the reciprocal of the Reynolds number, this is, $h_{m}=1 / R e$ for cylinder case. For airfoil case, coarser grid is enough to obtain convergence results and the reliability of the results need different grid resolution to test and compare.

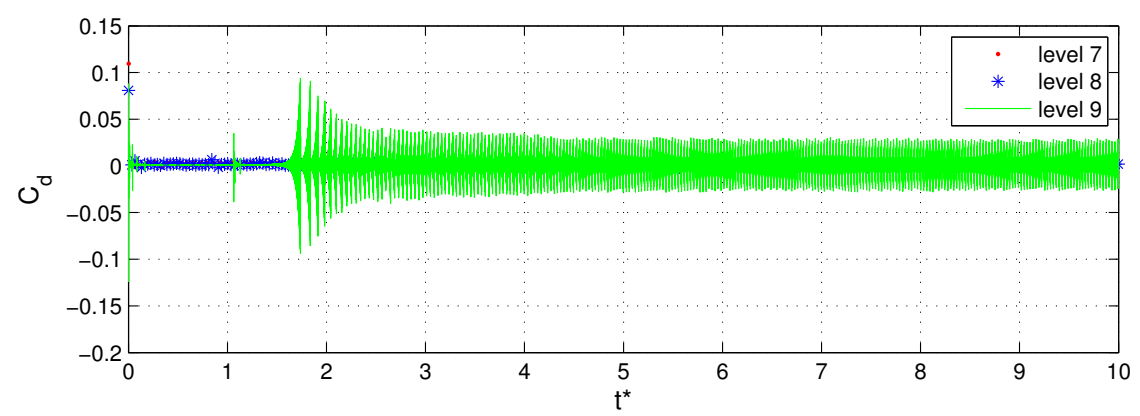

(a) Cylinder

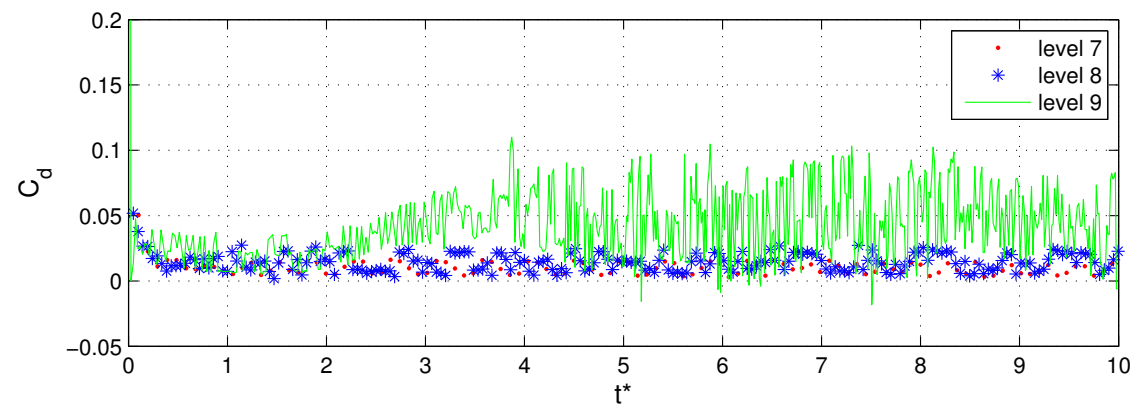

(b) Airfoil NACA2414

Figure 10: The drag force coefficient variations with time at $R e=1 \times 10^{5}$. 


\subsection{D computational results with free surface}

For 3D cases with water free surface, the bluff body is a floating sphere with half of it submerging into the water and the streamlined body is the series 60 ship hull. A few results are presented in this paper for the limited length of the paper.

The drag force variations with time evolving is shown in Figure 11(a) with grid levels 8 and 9 . The two grid level results show fair agreement and this means that the fluid field is convergent to a ideal one. The result of floating sphere is similar but the fluctuation is more violent for its bluff body, as shown in Figure 11(b).

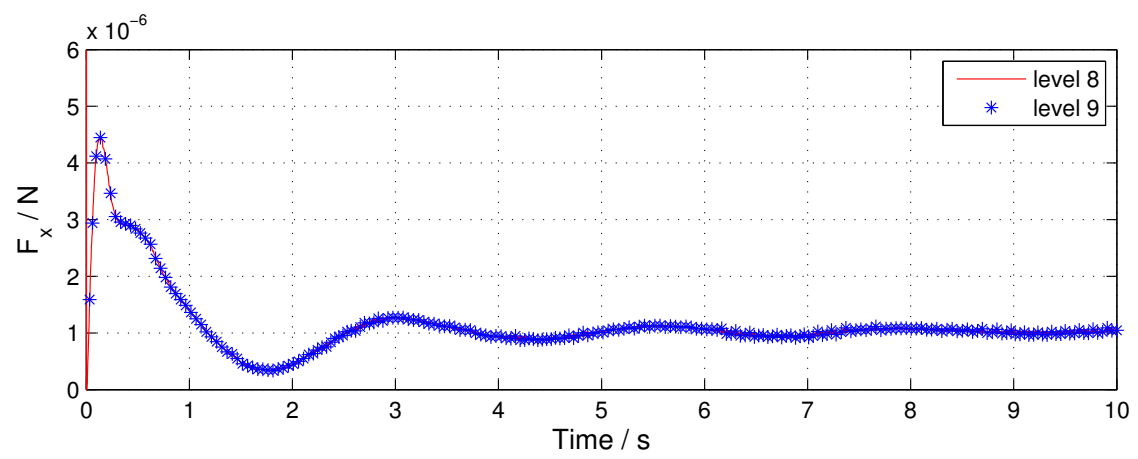

(a) Ship

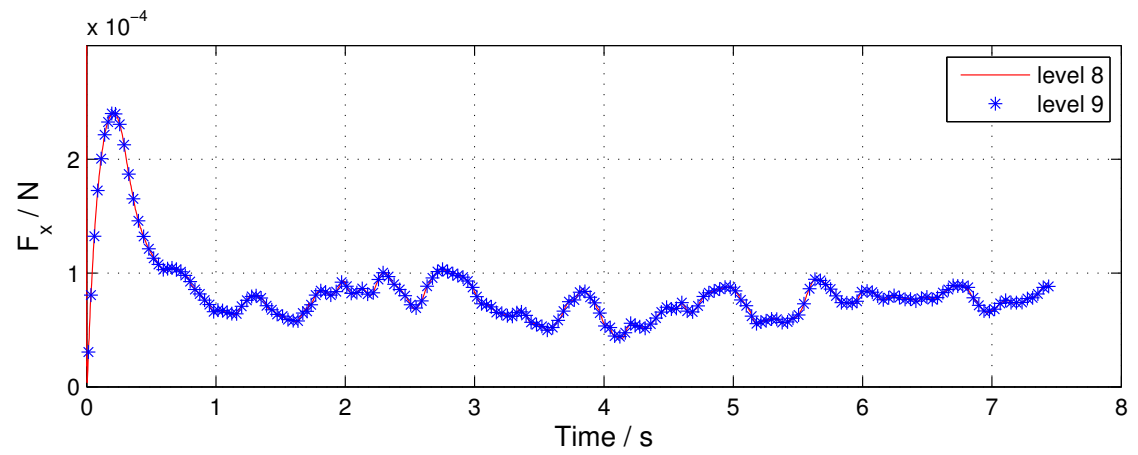

(b) Sphere

Figure 11: Drag force coefficient variations with different grid levels.

Moreover, the wave pattern in Figure 12 of ship hull is conformal with the literature ones $[18,19]$. For the streamlined floating body, e.g., the series 60 ship hull, the beautiful wave pattern is formed and the wave angle is not change with the time evolving. Therefore, the wave field can be regarded as steady one for streamlined floating body. But for bluff floating body, e.g., floating sphere, the wave pattern is not steady one, as shown in Figure 13 and so the drag force would present fluctuation, as shown in Figure 11(b). 


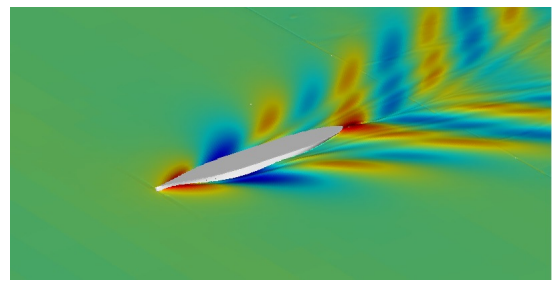

Figure 12: The wave pattern induced by ship.

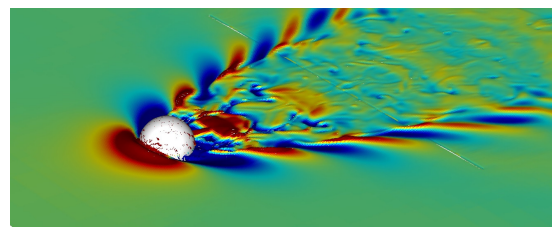

Figure 13: The wave pattern induced by floating sphere.

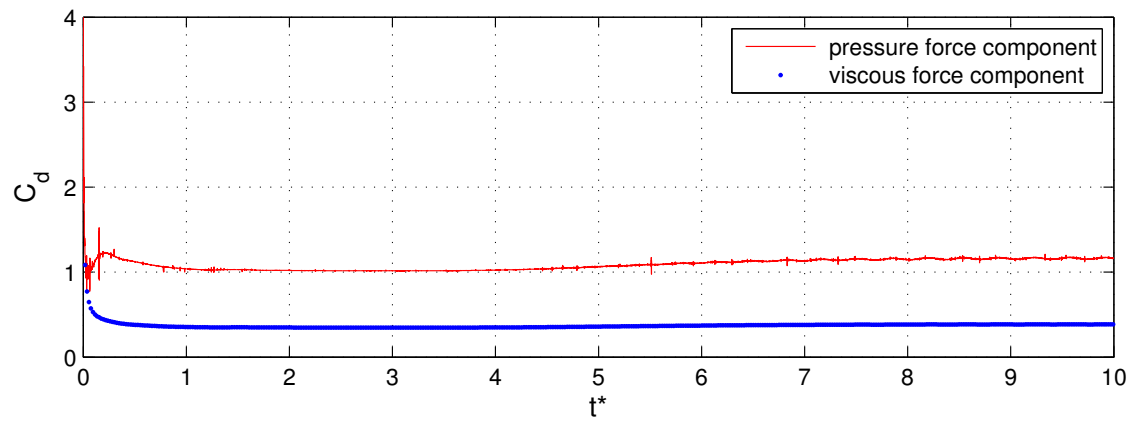

(a) Cylinder

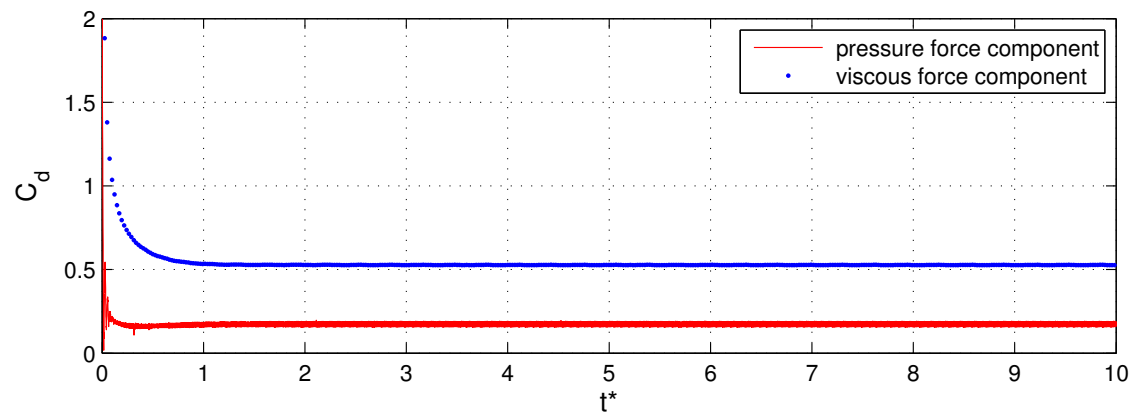

(b) Airfoil NACA2414

Figure 14: Pressure force and viscous force variations with time $R e=100$. 


\subsection{Fluctuations of pressure and viscous forces}

The drag force coefficient can be divided into two parts, pressure and viscous force components. And we analyze the relationship between the partial force with the grid resolution. For the cylinder and airfoil cases below, the grid level is equal to 9 without specific statement.

For the bluff body, e.g., cylinder in this paper, the pressure force component is lager than the viscous one for even with low Reynolds number, like as $R e=100$. The viscous force component stands a stable constance after it is convergent, but the pressure force component has fluctuation, as shown in Figure 14(a). For streamlined body, e.g., airfoil in this paper, the situation is opposite. This means that the viscous force component is lager than pressure one with $R e=100$, as shown in Figure 14(b).

With Reynolds number 1000 , the cylinder case does convergent to a stable value as stated previously. And the pressure force is lager and has more violent fluctuation than the viscous force, as shown in Figure 15(a). For airfoil case with the same Reynolds number, as shown in Figure 15(b), the viscous force component is approaching to pressure force and there is almost no fluctuation for both of them.

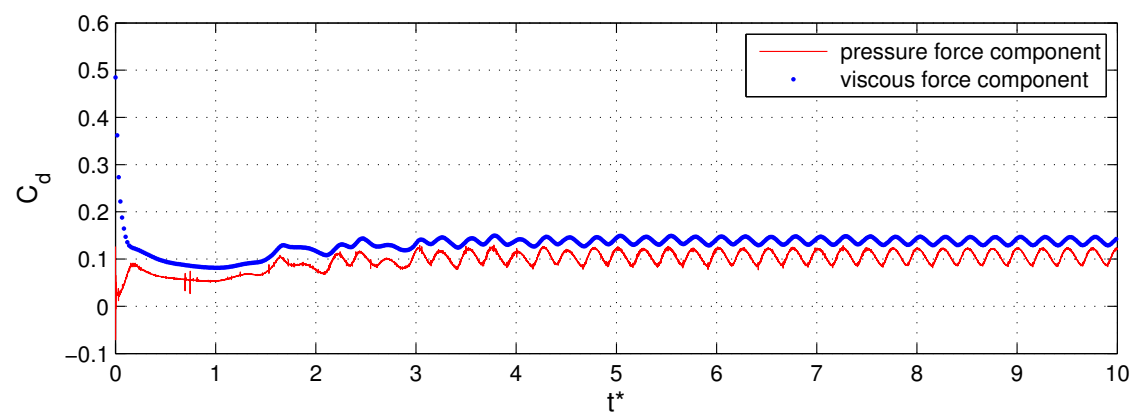

(a) Cylinder

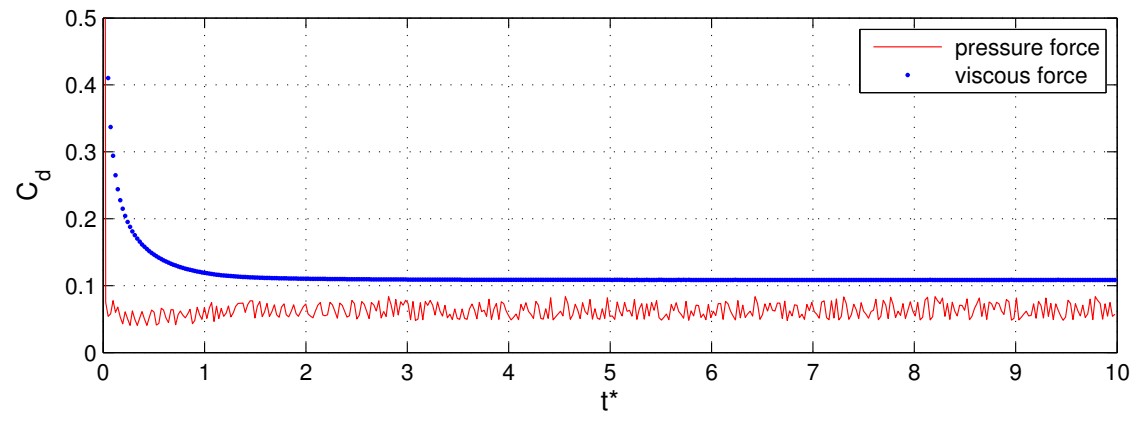

(b) Airfoil NACA2414

Figure 15: Pressure force and viscous force variations with time $R e=1000$. 


\section{Conclusion}

The validations of the fluid fields with bluff or streamlined body are carried out employing open CFD software Gerris. The criteria is based on the force coefficient convergence with different grid levels. The computational process shows that it easy to adjust the grid resolution for various grid levels of a certain boundary case with or without water free surface.

The results show that the fluid field with streamlined body without free surface is easier to converge than the bluff body. For the water surface cases, the force coefficient would converge to a ideal one for both bluff and streamlined bodies. The wave field of streamlined body would be regarded as steady one for a certain Froude number condition. But for the bluff body case, the force coefficient would present fluctuation with small amplitude. The convergence validation should be implemented preliminarily for the computation of the fluid dynamics and it is easy to do in Gerris just to modify the grid level parameter in the input script file.

\section{Acknowledgement}

This project is supported by the National Natural Science Foundation of China (Grant No. 51009147) and the Major State Basic Research Development Program of China (973 Program) (Grant No. 2014CB046801 and 2014CB046804).

\section{References}

[1] Popinet, S., Gerris: a tree-based adaptive solver for the incompressible Euler equations in complex geometries. Journal of Computational Physics, 190(2), pp. 572-600, 2003.

[2] Zhang, Z., Wieland, R., Reiche, M., Funk, R., Hoffmann, C., Li, Y. \& Sommer, M., Wind modelling for wind erosion research by open source computational fluid dynamics. Ecological Informatics, 6(5), pp. 316-324, 2011.

[3] An, H. \& Yu, S., Well-balanced shallow water flow simulation on quadtree cut cell grids. Advances in Water Resources, 39, pp. 60-70, 2012.

[4] Viroulet, S., Cebron, D., Kimmoun, O. \& Kharif, C., Shallow water waves generated by subaerial solid landslides. Geophysical Journal International, 193(2), pp. 747-762, 2013.

[5] Rickard, G., O'Callaghan, J. \& Popinet, S., Numerical simulations of internal solitary waves interacting with uniform slopes using an adaptive model. Ocean Modelling, 30(1), pp. 16-28, 2009.

[6] Wroniszewski, P.A., Verschaeve, J.C.G. \& Pedersen, G.K., Benchmarking of navier-stokes codes for free surface simulations by means of a solitary wave. Coastal Engineering, 91, pp. 1-17, 2014.

[7] Popinet, S., Quadtree-adaptive tsunami modelling. Ocean Dynamics, 61(9), pp. 1261-1285, 2011. 
[8] Popinet, S., Adaptive modelling of long-distance wave propagation and finescale flooding during the Tohoku tsunami. Natural Hazards and Earth System Sciences, 12(4), pp. 1213-1227, 2012.

[9] O'Callaghan, J., Rickard, G., Popinet, S. \& Stevens, C., Response of buoyant plumes to transient discharges investigated using an adaptive solver. Journal of Geophysical Research-Oceans, 115, 2010.

[10] Fuster, D., Agbaglah, G., Josserand, C., Popinet, S. \& Zaleski, S., Numerical simulation of droplets, bubbles and waves: state of the art. Fluid Dynamics Research, 41(6), 2009.

[11] Staron, L., Lagree, P.Y., Ray, P. \& Popinet, S., Scaling laws for the slumping of a bingham plastic fluid. Journal of Rheology, 57(4), pp. 1265-1280, 2013.

[12] Josserand, C., Lagree, P.Y., Lhuillier, D., Popinet, S., Ray, P. \& Staron, L., The spreading of a granular column from a Bingham point of view, volume 1145 of AIP Conference Proceedings, pp. 631-634, 2009.

[13] Lagree, P.Y., Staron, L. \& Popinet, S., The granular column collapse as a continuum: validity of a two-dimensional Navier-Stokes model with a mu(i)rheology. Journal of Fluid Mechanics, 686, pp. 378-408, 2011.

[14] Fuster, D., Bague, A., Boeck, T., Le Moyne, L., Leboissetier, A., Popinet, S., Ray, P., Scardovelli, R. \& Zaleski, S., Simulation of primary atomization with an octree adaptive mesh refinement and VOF method. International Journal of Multiphase Flow, 35(6), pp. 550-565, 2009.

[15] Popinet, S., Smith, M. \& Stevens, C., Experimental and numerical study of the turbulence characteristics of airflow around a research vessel. Journal of Atmospheric and Oceanic Technology, 21(10), pp. 1575-1589, 2004.

[16] Stringer, R.M., Zang, J. \& Hillis, A.J., Unsteady rans computations of flow around a circular cylinder for a wide range of reynolds numbers. Ocean Engineering, 87, pp. 1-9, 2014.

[17] Zhao, L.H., Liu, M. \& Lv, T., Study on the aerodynamic performance of blade airfoil of vertical axis wind turbine at low reynolds number. Information Technology Journal, 12(14), pp. 3042-3045, 2013.

[18] Simonsen, C.D. \& Stern, F., Rans maneuvering simulation of esso osaka with rudder and a body-force propeller. Journal of Ship Research, 49(2), pp. 98$120,2005$.

[19] Wanderley, J.B.V., Vitola, M., Sphaier, S.H. \& Levi, C., A three-dimensional numerical simulation of the free surface flow around a ship hull, InTech, pp. 395-408, 2011. 